\title{
Less is not always more: embracing (appropriate) medical intensity
}

Laura G Burke, ${ }^{1,2}$ Ashish K Jha ${ }^{1,3}$

${ }^{1}$ Department of Health Policy and Management, Harvard School of Public Health, Boston, Massachusetts, USA ${ }^{2}$ Department of Emergency Medicine, Beth Israel Deaconess Medical Center, Boston, Massachusetts, USA ${ }^{3}$ Division of General Internal Medicine, Brigham and Women's Hospital, Boston, Massachusetts, USA

\section{Correspondence to}

Dr Ashish K Jha, Department of Health Policy and Management and the Division of General Internal Medicine, Brigham and Women's Hospital (AKJ), Boston, MA 02115, USA;

ajha@hsph.harvard.edu

Received 16 September 2014 Accepted 17 September 2014 Published Online First

1 October 2014

\section{SLinked}

- http://dx.doi.org/10.1136/ bmjqs-2014-002816

\section{CrossMark}

To cite: Burke $L G$, Jha $A K$. BMJ Qual Saf 2014;23: 968-969.
As healthcare costs continue to rise globally, policy makers and researchers have focused on inefficiencies in healthcare delivery. In the USA, the combination of high costs and disappointing outcomes associated with a fragmented delivery system has generated the idea that substantial reductions in spending are possible without sacrificing patient care. Indeed, we know that high spending often fails to produce optimal outcomes and therefore, reductions in spending should be achievable with no detrimental effects on quality.

The evidence that less can sometimes be more is all around us. Front-line providers know all too well the human and financial costs of aggressive treatment of terminally ill patients who might otherwise prefer a palliative approach. Redundant testing due to inadequate care coordination and lack of information sharing is another example. Preventable adverse events exemplify how poorquality care can drive up costs and harm patients at the same time. From these instances, a new mantra has emerged among many health policy leaders: less is better. Intensity is bad.

What is clinical intensity? It is often described as the tendency to do more: perform more procedures, admit more patients, consult more specialists and prescribe more medications. ${ }^{1}$ Why does level of intensity appear to vary widely across institutions? Some have suggested that variation in clinical intensity is due to differences in the underlying patient population, although others have argued that patient factors matter very little. Detailed work by researchers at Dartmouth has suggested that both medical intensity and its variation among providers are fundamentally inefficient and are driven by differences in medical culture. ${ }^{2-4}$ The nature and value of medical intensity are controversial and ripe for additional research.
In this context, an elegant new study by Bhatia et al offers several important insights. The first is that providing less medical care (i.e. having lower intensity) has its own costs. The authors examined rates of admission from emergency departments (EDs) for patients presenting with heart failure (HF). They found that the low-intensity EDs, those with low baseline rates of admissions for patients with $\mathrm{HF}$, had higher rates of repeat ED visits and hospitalisations. Even more worrisome, low admission rate EDs had a trend toward greater mortality relative to institutions with higher admission rates for this condition. Patients discharged from EDs with low rates of admission were less likely to see a cardiology specialist or to have cardiac testing after discharge. Taken together, these findings suggest that there are real clinical costs to being less aggressive. Many patients might have done better had they gone to an ED with a higher level of clinical intensity.

A second insight from the Bhatia study is that intensity does seem to be related to clinical culture. While the authors found that low admission rate hospitals tended to be smaller, non-teaching, rural hospitals (with presumably healthier patients), these characteristics did not fully explain the differences in hospitalisations after presentation. Indeed, the authors found that when patients presented to a different hospital after being discharged from the original ED, their likelihood of admission on the second visit was predicted by the overall admission rate for the second hospital. This suggests that variations in admission rates are driven by differences in practice patterns rather than the degree of the patient's illness.

Clinicians who have worked at multiple institutions will not be surprised. Indeed, we are all familiar with the phenomenon of 'medical culture.' What is common practice at one hospital (e.g. discharging 
a patient with low-risk chest pain after a single set of cardiac enzymes) may be viewed as substandard or even reckless at another. It seems that this phenomenon of medical culture at least partly explains some of the variation in medical intensity among providers and institutions.

The study by Bhatia et al adds to a growing body of literature that challenges the 'less is more' philosophy in health policy. Their study is consistent with the other literature showing that aggressive, high-intensity care is associated with better outcomes for some conditions. ${ }^{6-8}$ The policy implication of this phenomenon is clear: even in health care, sometimes, 'you get what you pay for'. But how does one reconcile the findings of the Bhatia study and others like it with the Dartmouth work suggesting that higher spending does not produce better outcomes? Is medical intensity bad or is it good? Clearly, the answer depends on the specific clinical scenario. Patients and their conditions are too heterogeneous for a single approach. The optimal level of intensity of care needs to be tailored to the patient's needs and wishes and supported by highquality evidence whenever possible. Optimal care for patients with serious illness frequently requires complex care in expensive settings..$^{9} 10$ This level of intensity, applied to seriously ill patients, can lead to better outcomes. Applying the same level of intensity to a different patient population is likely to lead to waste and potentially worse clinical outcomes.

The recognition of waste and inefficiency has motivated policymakers in a number of countries to begin a shift away from a fee-for-service model, which often encourages volume over value. As this shift continues, we need to remember the corollary: that less is also not always better. This emerging body of work has important implications for policymakers; indiscriminate cuts in spending are unhelpful. A more nuanced approach to intensity and spending is necessary. While providing less medical care is guaranteed to reduce costs in the short run, it is likely to lead to poor outcomes for at least some conditions. Sophisticated approaches to measuring patient outcomes will be critical to ensuring that as payment models evolve, we reduce wasteful spending by eliminating use of services on patients who will not benefit-not by skimping on interventions that can save lives and improve health. This is the right kind of medical intensity.

Bhatia et al provide one more critically important insight; no one has quite gotten this right. While it is true that some patients at the high admission hospitals were likely admitted unnecessarily (i.e. they would have done fine at home), it is just as true-and quite problematic-that the low admission hospitals failed to admit patients who probably would have done better in the hospital. On average, the patients at the high admission hospitals seem to fare better, but patient care has to be about more than averages. New approaches to identifying, in real time, which patients should be admitted and which can go home safely are imperative. Just as importantly, we need to determine which services will foster a path to safe recovery at home for appropriately selected individuals. Right now, these decisions are based on local culture and custom. When medicine was a cottage industry, driven more by whims and opinions of eminent intellectuals, this kind of practice made sense. But as we shift towards being a scientific discipline, driven by evidence and data, this variation can no longer stand. Simplistic notions, such as doing more is better or doing less is better, have no role, either clinically or in the policy world. Figuring out who is likely to benefit from intensive therapy and who will do fine without is critically important-and not just for the bottom line. Indeed, patients' lives depend on it.

Contributors AKJ and LGB both made substantial contributions to the conception or design of the work, drafted the work and revised it critically for key intellectual content, approved the final version to be published and agreed to be accountable for all aspects of the work.

Competing interests None.

Provenance and peer review Not commissioned; internally peer reviewed.

\section{REFERENCES}

1 Sirovich BE, Gottlieb DJ, Welch HG, et al. Variation in the tendency of primary care physicians to intervene. Arch Intern Med 2005;165:2252-6.

2 Song Y, Skinner J, Bynum J, et al. Regional variations in diagnostic practices. N Engl J Med 2010;363:45-53.

3 Mittler JN, Landon BE, Fisher ES, et al. Market variations in intensity of Medicare service use and beneficiary experiences with care. Health Serv Res 2010;45:647-69.

4 Sirovich B, Gallagher PM, Wennberg DE, et al. Discretionary decision making by primary care physicians and the cost of $U$. S. Health care. Health Aff (Millwood) 2008;27:813-23.

5 Bhatia RS, Austin PC, Stukel TA, et al. Outcomes in patients with heart failure treated in hospitals with varying admission rates: population-based cohort study. BMJ Qual Saf 2014;23:981-8.

6 Silber JH, Kaestner R, Even-Shoshan O, et al. Aggressive treatment style and surgical outcomes. Health Serv Res 2010;45(6 Pt 2):1872-92.

7 Romley JA, Jena AB, Goldman DP. Hospital spending and inpatient mortality: evidence from California: an observational study. Ann Intern Med 2011;154:160-7.

8 Stukel TA, Fisher ES, Alter DA, et al. Association of hospital spending intensity with mortality and readmission rates in Ontario hospitals. JAMA 2012;307:1037-45.

9 Joynt KE, Orav EJ, Jha AK. The association between hospital volume and processes, outcomes, and costs of care for congestive heart failure. Ann Intern Med 2011;154:94-102.

10 McCrum ML, Lipsitz SR, Berry WR, et al. Beyond volume: does hospital complexity matter? an analysis of inpatient surgical mortality in the United States. Med Care 2014;52:235-42. 